a positive FOBT result are referred for colonoscopy, which puts great pressure on already stretched resources. As $80 \%$ of positive FOBT results are false, CT colonography (CTC) has been suggested as a less invasive alternative to colonoscopy.

To assess the value of CTC as a replacement test for colonoscopy in patients with a positive FOBT result, Walleser et al. performed a systematic review of five eligible studies to compare CTC with colonoscopy for sensitivity and specificity and to assess the cost-effectiveness of the two procedures. CTC detected $63 \%$ of lesions $10 \mathrm{~mm}$ or larger in diameter, but colonoscopy was significantly more sensitive, detecting $95 \%$ of such lesions. The specificity of colonoscopy was also higher at $99.8 \%$, compared with $95 \%$ for CTC. Colonoscopy was more sensitive (96\%) and more specific $(99.7 \%)$ than CTC (89\% and $96 \%$, respectively) for detecting the presence of any cancerous lesion.

Taking costs into account, the authors conclude that CTC is, overall, less effective, less accurate and more costly than colonoscopy in patients with a positive FOBT, primarily owing to better accuracy with colonoscopy. They suggest, however, that the use of CTC has future potential as the technology is evolving and accuracy might improve.

Original article Walleser S et al. (2007) What is the value of computered tomography colonography in patients screening positive for fecal occult blood? A systematic review and economic evaluation. Clin Gastroenterol Hepatol 5: $1439-1446$

\section{Repeat liver transplantation is possible in cases of aggressive HCV infection}

Patients who develop recurring cholestatic HCV infection after liver transplantation often experience graft failure. Most transplant centers exclude such patients from repeat transplants because of their extremely poor prognosis; however, in this report, Kwo et al. describe two patients with an aggressive HCV infection whose second liver transplant proved successful due to the administration of interferon therapy during the course of surgery.

Both patients had developed a clinically aggressive HCV infection after the initial liver transplants. Infection in the first patient developed when standard interferon and ribavirin therapy were discontinued due to nonresponse. The second patient developed HCV recurrence with cholestasis 2 months after an apparently successful graft. In both patients, after therapy with interferon $a-2 b$ and ribavirin, $\mathrm{HCV}$ was undetectable and repeat orthotopic liver transplantation was performed. Interferon $a-2 b$ was administered intravenously in both patients from the first incision to the end of surgery ( $4 \mathrm{~h})$. This approach was intended to produce high serum interferon levels to prevent any possibility of HCV re-infection in the new graft. Both patients recovered well, and no evidence of recurrent viremia was reported at 36 and 24 months, respectively.

The authors conclude that aggressive therapy with interferon and ribavirin can achieve HCV RNA clearance in patients with cholestatic post-transplant HCV infection, enabling a successful repeat transplant. They recommend further investigation of the anhepatic administration of interferon to liver transplant patients at high risk of HCV recurrence who can clear virus before repeat transplantation.

Original article Kwo PY et al. (2007) Intravenous interferon during the anhepatic phase of liver retransplantation and prevention of recurrence of cholestatic hepatitis $C$ virus. Liver Transpl 13: 1710-1713

\section{Passive immunotherapy as a vaccine against hepatitis $C$}

The diversity of HCV makes immunotherapy or vaccine design very difficult. Law et al. have identified broadly neutralizing antibodies from a human with hepatitis $C$ and demonstrated their HCV-targeting activity in mice with a chimeric human-mouse liver.

Human monoclonal antibodies (mAbs), which showed binding specificity to three different antigenic regions on HCV E2 glycoprotein, were isolated. So-called antigenic region 3 (AR3)-specific antibodies were found to target more different strains of HCV in vitro than antibodies specific for antigenic regions 1 and 2 . This observation suggests that AR3 is a highly conserved region, making it an ideal target for passive immunotherapy. To determine whether or not the AR3-specific antibodies could protect against $\mathrm{HCV}$ infection in vivo, they were given to mice genetically engineered to support a large population of human liver cells. Nine 